\title{
1 Do persistent rare species experience stronger negative frequency dependence than
}

\section{2 common species?}

3 Glenda Yenni ${ }^{1,}$ *, Peter B. Adler ${ }^{2}$, S. K. Morgan Ernest ${ }^{1}$

$4{ }^{1}$ Department of Wildlife Ecology and Conservation, University of Florida, Gainesville, FL

$5 \quad 32611$

$6 \quad{ }^{2}$ Department of Wildland Resources, Utah State University, Logan, UT, 84322

7 *Corresponding Author: Glenda M. Yenni, Department of Wildlife Ecology and Conservation,

8 University of Florida, 110 Newins-Ziegler Hall, PO Box 110430, Gainesville, FL 32611

9435213 6860,glenda@weecology.org

10 Keywords: rare species, coexistence, frequency dependence

11 Running Title: Asymmetric NFD and persistent rare species

12 Abstract: 216 words

13 Body: 4012 words

14 References: 27 


\section{Abstract}

18 challenge for both theoretical and empirical ecologists. Yenni, Adler, and Ernest (2012) proposed

19 that strong negative frequency dependence causes species to be rare while simultaneously

20 buffering them against extinction. This hypothesis predicts that, on average, rare species should

21 experience stronger negative frequency dependence than common species. However, it is

22 unknown if ecological communities generally show this theoretical pattern, or if rarity is

23 primarily determined by other processes that overwhelm the effects of strong negative frequency

24 dependence. We discuss the implications of this mechanism for natural communities, and

25 develop a method to test for a non-random relationship between negative frequency dependence

26 and relative abundance, using species abundance data from 90 communities across a broad range

27 of environments and taxonomic groups. To account for biases introduced by measurement error,

28 we compared the observed correlation between species relative abundance and the strength of

29 frequency dependence against expectations from a randomization procedure. In approximately

30 half of the analyzed communities, rare species showed disproportionately strong negative

31 frequency dependence compared to common species. Specifically, we found a pattern of

32 increasingly strong negative frequency dependence with decreasing relative abundance. Our

33 results suggest that strong negative frequency dependence is a signature of both rarity and

34 persistence for many species in many communities.

\section{Introduction}

36 Rare species are ubiquitous in nature, but understanding the factors limiting their

37 abundance and the mechanisms allowing them to persist has proven challenging. Reproducing 
realistic local-scale community structures with numerous rare species remains difficult using simple theoretical or statistical models of species interactions (Tilman, 2004; Angert et al., 2009;

Clark et al., 2010). One reason these models fail to predict realistic numbers of rare species is that many rare species are simply ephemeral, and their occasional presence is likely explained by metapopulation dynamics. Thus the number of these species in a local community can only be explained when regional-scale processes are considered along with local-scale processes.

However, not all rare species are ephemeral. Some species manage to persist at low abundance within a community for long periods of time without succumbing to stochastic extinction events.

Since stochasticity is pervasive in ecological systems, species with low population sizes need mechanisms that buffer their population dynamics from extinction in order to persist (Tilman, 2004; Kobe \& Vriesendorp, 2011). It is this subset of rare species, the persistent rare species, which remain a paradox for coexistence. Despite the importance of understanding how these rare species are able to persist, how to determine a rare species' role in a community as persistent or ephemeral, how prevalent persistent rare species are, and what allows their persistence all remain controversial (Preston, 1948; Main, 1982; Kunin \& Gaston, 1997; Magurran \& Henderson, 2003;

53 Comita et al., 2010). Here we provide empirical evidence for a recently proposed mechanism

54 that, in theory, can buffer small populations against extinction: asymmetric negative frequency dependence (Yenni et al., 2012).

\section{The role of Negative Frequency Dependence in rarity and persistence}


61 et al., 2007). Negative frequency dependence occurs when a species' population growth rate is

62 more strongly limited by the density of conspecifics than by the density of heterospecifics (Fig

63 1A). When negative frequency dependence is strong, relatively small increases in a species'

64 density, and in turn its frequency in the community, will result in large declines in population

65 growth rate (Fig 1B). Holding the maximum population growth rate constant, species with

66 stronger negative frequency dependence must have lower relative abundance than species with

67 weak negative frequency dependence. In other words, for species experiencing strong negative

68 frequency dependence, intraspecific competition is the primary cause of rarity (Appendix S2 in

69 Supporting Information).

70 In contrast, studies of competition often assume that rare species are primarily suppressed

71 by interspecific competition (Chesson, 2000; Tilman, 2004). But if interspecific competition is

72 the cause of rarity, how can we explain the long-term persistence of many rare species? Why

73 don't the superior competitors exclude rare species altogether? Our alternative explanation, that

74 rare species are strongly frequency-dependent, also explains persistence, because strong

75 population regulation buffers species against extinction. It is initially counter-intuitive that a

76 process causing rarity could also promote persistence. However, when a species with strong

77 negative frequency dependence drops below its equilibrium frequency, it will experience a large

78 increase in population growth rate (Fig. 1). Because species with strong negative frequency

79 dependence are more sensitive to increases in their own population numbers, they experience

80 relatively weak suppression by dominant community members; they are effectively released

81 from competitive effects as their densities approach zero. Thus, strong negative frequency

82 dependence can simultaneously limit species' average abundances while buffering them against

83 extinction if they fall to low density (Yenni et al., 2012). 

species (Yenni et al., 2012). Common species are less vulnerable to stochastic extinction due to their higher abundances. While common species might experience strong or weak negative

87 frequency dependence, rare species lacking strong negative frequency dependence would be quickly lost from a local community due to stochastic extinction. In addition, if a rare and common species experience the same degree of negative frequency dependence (equivalent slopes in Fig 1), then the rare species must have a lower maximal growth rate. Holding the equilibrium abundances constant, for the rare species to experience the same buffering against stochastic extinction (i.e. similar maximal growth rates), it must have stronger negative

94 (frequency) and the strength of negative frequency dependence: species with lower relative 95 abundances should experience stronger negative frequency dependence.

\section{The Asymmetric Negative Frequency Dependence Pattern}

While simulations suggest that strong negative frequency dependence should be a common feature of persistent rare species (Yenni et al., 2012), few empirical studies have assessed this mechanism. Strong negative density or frequency dependence in some rare species has been documented (Harpole \& Suding, 2007; Adler et al., 2010; Comita et al., 2010; Mangan et al., 2010; Johnson et al., 2012; Xu et al., 2015) but these few studies exclusively focused on select

102 plant communities. If this is a general pattern across taxa and ecosystems, then this mechanism

103 could be of general importance to the coexistence and persistence of rare species. However, this

104 limited number of studies does not tell us how general this pattern is across a variety of natural

105 communities. Testing the asymmetric negative frequency dependence hypothesis is challenging 
107 dependence to buffer species against stochastic extinction. However, we can examine whether

108 rarer species have stronger negative frequency dependence than more common species, which is

109 equivalent to testing whether rare species are more sensitive to intra- than interspecific

110 competition when compared to common species. If strong negative frequency dependence is

111 more common among rare species, it would suggest 1) that rare species are not rare because they

112 are suppressed by superior competitors, but rather because they are more strongly self-limiting,

113 and 2) that rare species that do not experience a large release from competition at low density, as

114 implied by strong negative frequency dependence, often do not persist.

\section{Testing for Asymmetric Negative Frequency Dependence}

116 To explore the relationship between rarity and negative frequency dependence in

117 ecological communities, we decided to assess whether there is a general relationship between the

118 rarity of a species and the strength of negative frequency dependence by examining a large

119 compilation of publicly available time-series on the abundance of all species within one trophic

120 guild. Using this approach to evaluate the relationship between abundance and rarity requires 1)

121 identification of appropriate data, 2) estimating equilibrium frequency and negative frequency

122 dependence for each species in each community, 3) identifying the persistent community, 4)

123 estimating a community-level pattern of negative frequency dependence, and 5) accounting for

124 known and unknown biases in the pattern of interest before making a final conclusion about the

125 negative frequency dependence-rarity relationship in each community. The methods we

126 developed to deal with these issues have allowed us to use commonly available data to begin to

127 explore the relationship between negative frequency dependence and rarity. We hope our results

128 will spur future research and collection of even more useful data. 


\section{Identifying Suitable Data for Community Negative Frequency Dependence Patterns} operate at very small spatial scales (Chesson, 2000). This is also true in the framework defined in

132 Yenni et al. (2012). Therefore, to assess whether persistent rare species have stronger negative

133 frequency dependence than common species, we required communities where the data was

134 collected in a manner consistent with this local-scale concept. We restricted communities to all

135 species within a study belonging to the same trophic level and occurring within a single habitat

136 type as defined by the data collector. If the data collector indicated that the study included

137 multiple sites of different habitat types or multiple trophic levels these data were treated as

138 different communities and analyzed separately. If a study collected data from multiple locations

139 of the same habitat type, we examined those separately. However, to keep a single study area

140 from dominating the results because it had multiple sites, we treated multiple sites in a single

141 study area as habitat replicates and only one covariance was estimated from the entire resulting

142 site data. Finally, to calculate frequency dependence, we used time series data because studies

143 measuring frequency dependence for all species in a community in an experimental manner are

144 rare. All of these data decisions were made to ensure we were only analyzing populations that

145 were likely to be directly interacting and to provide data for a large number and diversity of taxa

146 and ecosystems. Using these criteria, we found suitable data for 90 different communities

147 containing collectively 703 species (Appendix S1 in Supplementary Information). These

148 communities represent six major taxonomic groups (birds, fish, herpetofauna, invertebrates,

149 mammals, and plants), 5 continents, and 3 trophic levels, thus providing a general exploration of

150 whether persistent rare species commonly exhibit stronger negative frequency dependence, and

151 thus, stronger population buffering, than common species. 


\section{Calculating Negative Frequency Dependence and Equilibrium Frequency}

156 We estimated a species' negative frequency dependence as the linear relationship between its

157 relative abundance (frequency) in a community and its annual per capita population growth rate.

158 For each community, relative abundance in each year $t$ for each species $s$ was calculated as $\mathrm{x}_{t, s}=$

$159 \mathrm{~N}_{t, s} / \Sigma_{\mathrm{s}=1: \mathrm{S}} \mathrm{N}_{t, s}$ (where $\mathrm{N}$ is a species' absolute abundance). Log per capita population growth

160 rates in each year $t$ for each species $s$ were calculated as $\mathrm{y}_{t, s}=\log \left(\mathrm{N}_{t+1, s} / \mathrm{N}_{t, s}\right)$. The relationship

161 between these population parameters was described as $\mathbf{Y}_{s}=\beta_{0, \mathrm{~s}}+\beta_{1, \mathrm{~s}} \mathbf{X}_{s}+\boldsymbol{\varepsilon}_{s}$ for each species $s$.

162 Equilibrium frequency $(f)$, a species' expected relative abundance in the community, is estimated

163 as the $\mathrm{x}$-intercept of this linear relationship, $f=-\beta_{0, \mathrm{~s}} / \beta_{1, \mathrm{~s}}$. Frequency dependence $(F D)$ is

164 estimated as the slope of the linear relationship, $F D=\beta_{1, \mathrm{~s}}$.

\section{Ephemeral vs. Persistent Species}

As discussed in the introduction, asymmetric negative frequency dependence is a

167 mechanism for maintaining small populations near their steady state despite stochasticity, and

168 thus only applies to stably coexisting common and rare species. Because other mechanisms are

169 expected to dominate the population dynamics of invading, declining, or transient species, we

170 used three criteria to identify and exclude species with population characteristics that would

171 make them likely to be ephemeral and not stably coexisting (Supplementary Figure S15): 1) too

172 few occurrences to estimate parameters (i.e. few or no adjacent non-zero abundances, the

173 inclusion of this type of species in a dataset is typically an arbitrary decision by the data 
174 collectors, and was highly variable between datasets), which indicates a species is likely

175 transient, 2) negative equilibrium frequency (Fig 1B), which indicates the species cannot persist

176 in the community (7.7\% of species, (Chesson, 2000; Adler et al., 2007)), and 3) positive

177 frequency dependence (Fig 1B), which is related to a negative invasion growth rate and likely

178 results in either the extinction of that species or an ever increasing invader $(11 \%$ of species,

179 (Chesson, 2000; Adler et al., 2007)). Because equilibrium frequency is estimated as the x-

180 intercept of the fitted relationship between log per capita growth rate and relative abundance, it is

181 possible for this value to be $<0$ or $>1$ (Fig 1B). Besides the fact that these species are obviously

182 ephemeral and thus irrelevant to our analysis, it would make the relationship between

183 equilibrium frequency and frequency dependence nonsensical to include negative equilibrium

184 frequencies or positive frequency dependences.

185 All other species are retained as persistent community members. Our criteria are only a

186 weak filter for removing ephemeral species. Some of the species retained in the analysis are

187 likely also ephemeral, but we abstained from further filtering to avoid possibly biasing the

188 results. We examined how this method of identifying persistent species relates to actual

189 persistence at a site by calculating the percent of years in which a species has non-zero

190 abundance and comparing these values between ephemeral and persistent species. Our approach

191 identified a group of more persistent species that were on average present over a higher fraction

192 of the time series (Supplementary Information Figure S14).

193 Quantifying Asymmetric Negative Frequency Dependence at the Community Level

194 We currently have no way to objectively characterize a species as experiencing weak or

195 strong negative frequency dependence. We can, however, estimate the relationship between

196 relative abundance and negative frequency dependence across all persistent species in the 
197 community. To quantify the relationship between rarity and negative frequency dependence

198 within a community, we calculated the covariance between equilibrium frequency and strength

199 of negative frequency dependence (Fig 2B). The covariance calculation uses every species in a

200 community as a single data point in the calculation (Fig $2 \mathrm{~B}): \operatorname{cov}(\log (\boldsymbol{f}), \log (-\boldsymbol{F D}))$. Negative

201 covariances indicate communities in which rare species are experiencing stronger negative

202 frequency dependence than their dominant counterparts (note that the expectation for $\operatorname{cov}(\log (f)$,

$203 \log (-\boldsymbol{F D}))$ is already negative, we discuss how we addressed this in the following section). Log-

$204 \log$ relationships were most appropriate for calculating these covariances because most

205 communities contain many species with very low equilibrium frequencies and only a few

206 dominants, and because the observed negative frequency dependence of the few dominants was

207 typically at least an order of magnitude lower than the remainder of the community.

208 Dealing with Uncertainty in Abundance Estimates

Time series data with significant observation uncertainty can show the signature of

210 negative density or frequency dependence even when none exists (Freckleton et al., 2006; Knape

$211 \&$ de Valpine, 2012) because the calculation can create a negative bias in the resulting population

212 growth estimates. We designed a randomization procedure to determine which communities

213 showed a significant negative covariance between equilibrium frequency and the strength of

214 negative frequency dependence. We reshuffled abundances from the original community time

215 series 5000 times for each species independently, and then repeated all estimation methods with

216 the time-randomized data. This procedure maintains relative abundances and observed

217 variability, but the frequency dependence detected in the randomized data is due to uncertainty

218 alone. We compared the covariance estimated from the original data to the distribution of

219 covariance estimated from the randomized data sets to estimate effect size and p-values. We 
220 report the difference in the observed pattern from the mean randomized pattern and calculate the

221 p-value as the proportion of randomized pattern values that are less than or equal to the observed

222 pattern. Simulations confirm that this approach accounts for known biases resulting from

223 uncertainty in the observations, as well as sampling biases for rare species (see Supplementary

224 Information for details). In addition to removing uncertainty bias, this has the added benefit of

225 removing any effects of community structure (e.g. richness) that may create a bias in the pattern

226 of interest, because it creates a randomized expectation for each community. This is a

227 conservative approach, but it enables us to identify communities in which the observed

228 relationship between rarity and NDF is extremely unlikely to arise due to chance or statistical

229 artifacts. The significance of the asymmetric negative frequency dependence pattern was

230 assessed using Strimmer's approach (Strimmer, 2008) to estimate the number of true null

231 hypotheses, controlling the false discovery rate at 0.1 (Benjamini \& Hochberg, 1995, 2000;

232 Verhoeven et al., 2005). All analyses were performed in the R platform ((R Development Core

233 Team, 2012) see Supplementary Information).

\section{Immigration or Competition?}

While Yenni et al (2012) predicted asymmetric frequency dependence emerging from competition, it is possible that other mechanisms could generate the same pattern. While, only competition has been explored theoretically, it is possible to imagine scenarios where other the more likely processes to generate asymmetric frequency dependence: immigration.

241 Brown, 1977) and is expected to have particular influence on populations of rare species, thus

242 creating the potential for immigration to influence or even create patterns of asymmetric 
243 frequency dependence. We used both our NFD simulation and a more traditional Lotka-Volterra

244 based model (Chesson, 2000) to explore how immigration influenced negative frequency

245 dependence in common and rare species (Appendix S4). Results from both models were similar:

246 immigration did not create patterns of asymmetric frequency dependence and, if anything,

247 significant amounts of immigration decreases the ability of our method to detect true in situ

248 asymmetric negative frequency dependence (models and results in Appendix S4).

\section{Asymmetric Patterns of Negative Frequency Dependence Appear to be Common}

When compared to the randomized time series, $46 \%$ (41 of 90 ) of communities

251

252

253

254

255

256

257

258

259

260

261

262

263

264

demonstrated a significant relationship between relative abundance and negative frequency

dependence, indicating that rare species experience stronger negative frequency dependence than common species (Fig 3A, Table 1). While negative relationships between rarity and the strength of negative frequency dependence often arose in randomized data as well (Fig 2B, 2C), in the communities where the observed pattern was statistically significant, the stronger negative frequency dependence of rare species could not be explained solely as a result of bias related to uncertainty in the abundance estimates. These results indicate real differences between rare and common species in the sensitivity of population growth rates to the abundance of heterospecifics and conspecifics.

The prevalence of asymmetric negative frequency dependence within communities varied by taxonomic group (Fig 3B-G). In approximately half of bird, fish, invertebrate, mammal and plant communities $(50 \%, 50 \%, 48 \%, 53 \%$ and 53\%, respectively) rare species experienced stronger negative frequency dependence than their dominant counterparts (Table 1, Fig 3). However, only $9 \%$ of herpetofauna communities had a significant asymmetric negative 
265 frequency dependence structure. Why asymmetric negative frequency dependence is less

266 prevalent in herpetofauna communities is unclear, though the documented sensitivity of

267 herpetofauna to disturbance is one possibility for their differing dynamics (Collins \& Storfer,

268 2003). Across all taxa, only a few communities had dominant species with stronger negative

269 frequency dependence estimates than the rare species, but this pattern was never significant (see

270 Table S1 in Supplementary Information). Thus, while rare species often, but not always, showed

271 significantly stronger negative frequency dependence than common species, common species

272 never showed stronger negative frequency dependence than rare species. Despite differences in

273 diversity, complexity of species interactions and how species may achieve the necessary

274 population dynamics, the phenomenon of rare species exhibiting stronger negative frequency

275 dependence than more common species appears to be widespread across community types.

Simulations testing our randomization procedure for addressing bias caused by measurement

277 error (Supplementary Information) demonstrated that our method consistently assigns

278 significance when the relationship between equilibrium frequency and negative frequency

279 dependence is strong, but it typically fails to detect weak relationships. Therefore, our non-

280 significant results likely include some communities where a relationship between relative

281 abundance and negative frequency dependence exists, but is too weak for our method to detect.

282 If so, then the prevalence of strong negative frequency dependence in rare species is likely more

283 common than our results indicate. Overall, our results suggest that in many communities,

284 persistent rare species may commonly exhibit stronger negative frequency dependence than the

285 more common species in the community.

\section{Implications}


The theory developed in Yenni et al. (2012) and the results presented here imply that

288 differences between rare and common species in their sensitivity to intraspecific and interspecific

289 competition may explain the existence of persistent rare species in many communities. This

290 finding leads to a variety of interesting research questions. Why are rare species more sensitive

291 to intraspecific than interspecific competition? One possibility is that both strong sensitivity to

292 congeners and an ability to increase rapidly when the population falls below its equilibrium

293 frequency are traits of rare species, e.g. a result of specialization on a rare resource or

294 susceptibility to virulent species-specific natural enemies. An alternative explanation is that

295 strong negative frequency dependence is not a fixed trait but is context-dependent. Some species

296 might show strong negative frequency dependence when conditions cause them to be rare

297 members of the community but weaker negative frequency dependence in communities when

298 they are more dominant. The limited availability of community time series data does not allow us

299 to directly link strong negative frequency dependence to species traits or to test whether some

300 species may show weak negative frequency dependence when they are dominant but strong

301 negative frequency dependence when they are rare.

Yenni et al (2012) described a theoretical framework in which the stronger negative

303 frequency dependence of rare species emerges from intraspecific competition. Our empirical

304 results are consistent with the theory and suggest that asymmetric density dependence may

305 structure many communities. Our simulations, provided in the Supplementary Information,

306 further support the idea that the prevalence of asymmetric negative frequency dependence is not

307 merely an artifact of uncertainty due to sampling biases or immigration. That is not to say that

308 immigration as an ecological process, along with other processes such as facilitation and

309 interaction networks, cycles, chaotic dynamics, and nonlinear negative frequency dependences 
310 could not also create or modify patterns of negative frequency dependence. For example, while

311 we explored a particular implementation of immigration, it is still possible that immigration

312 might be able to generate asymmetric negative frequency dependence under specific

313 circumstances. Clearly it is important for coexistence theory to assess whether these other

314 processes can also generate or enhance community-level patterns of asymmetric frequency

315 dependence, especially as it relates to the concept of persistent rare species.

316 Regardless of the underlying mechanisms, or whether strong negative frequency

317 dependence is a fixed or plastic trait of rare species, our results suggest that exploring the causes

318 and consequences of asymmetric negative frequency dependence may be important not only for

319 coexistence theory, but also for designing management strategies for rare species. If intraspecific

320 competition is the primary cause of rarity, then indirect effects of environmental change

321 mediated by competitive interactions (Adler et al., 2012) might be relatively weak for rare

322 species compared to common species. Similarly, we might expect management actions intended

323 to increase the abundance of a rare species by reducing the density of interspecific competitors to

324 be unsuccessful. However, if asymmetric negative frequency dependence is being generated by

325 other mechanisms such as immigration, then the implications for designing management

326 strategies will be very different. Investigating the mechanisms that lead to asymmetric negative

327 frequency dependence is an important step for understanding, and potentially managing, the

328 many rare species in natural communities.

\section{Future Analyses}

Our analysis also highlights analytical and theoretical limitations to our understanding of

331 negative frequency dependence that need to be addressed for research into negative frequency 
332 dependence to progress further. Definitive statements about the absolute strength of negative

333 frequency dependence in common and rare species would require unbiased estimates of

334 maximum population growth rates and density dependence. In contrast, our randomization test

335 focuses on the relative strength of negative frequency dependence in common vs. rare species.

336 Direct, unbiased estimates of negative frequency dependence would remove the need for our

337 conservative approach, and could reveal the role of asymmetric negative frequency dependence

338 in additional communities. An additional challenge for future research is disentangling the effects

339 of intra- and interspecific competition from other potential mechanisms of negative frequency

340 dependence such as immigration or nonlinear dynamics. Addressing this challenge will also

341 require more sophisticated methods for correcting negative frequency dependence for bias or,

342 alternatively, individual-level data on vital rates and dispersal.

\section{Summary}

Our work suggests that strong negative frequency dependence in persistent rare species could be a mechanism that helps offset stochastic extinction risk. If true, this concept has wideranging implications for our understanding of coexistence. However, in exploring this idea, we found severe limitations in the current state of data, statistics, and theory that prevented us from conducting robust empirical analyses or interpreting rigorously the mechanism behind the

349 patterns we uncovered. Given the importance, and possible generality, of asymmetric frequency

350 dependence, we hope that our work here will inspire others to help solve these challenges so we

351 may all better understand the mechanisms that allow rare species to persist in nature. 


\section{Acknowledgements}

355

A review by Dr. Robert Holt significantly improved the quality of this manuscript. Much

356 of the credit for the Supplementary Information on negative frequency dependence goes to Dr.

357 Holt. We thank all the researchers who have made their data publicly available and have

358 permitted its use in this manuscript. Further information about these datasets and dataset specific

359 acknowledgements of personnel and funding sources can be found in Appendix S1. Thank you to

360 Sarah Supp and the Mammal Community Database, Elita Baldridge, Ryan O'Donnell, and all the

361 contributors to the Ecological Data Wiki for assistance in identifying available community

362 datasets. G. Yenni was supported by the Ecology Center at Utah State University and an NSF

363 grant (DEB-1100664). PBA was supported by NSF DEB-1054040. 
365

366

367

368

369

370

371

372

373

374

375

376

377

378

379

380

381

382

383

384

385

386

387

388

389

390

391

392

393

394

395

References

Adler, P.B., Dalgleish, H.J. \& Ellner, S.P. (2012) Forecasting plant community impacts of climate variability and change: when do competitive interactions matter? Journal of Ecology, 100, 478-487.

Adler, P.B., Ellner, S.P. \& Levine, J.M. (2010) Coexistence of perennial plants: an embarrassment of niches. Ecology Letters, 13, 1019-1029.

Adler, P.B., HilleRisLambers, J. \& Levine, J.M. (2007) A niche for neutrality. Ecology Letters, 10, 95-104.

Angert, A.L., Huxman, T.E., Chesson, P. \& Venable, D.L. (2009) Functional tradeoffs determine species coexistence via the storage effect. Proceedings of the National Academy of Sciences of the United States of America, 106, 11641-11645.

Benjamini, Y. \& Hochberg, Y. (1995) Controlling the False Discovery Rate: A Practical and Powerful Approach to Multiple Testing. Journal of the Royal Statistical Society. Series B (Methodological), 57, 289-300.

Benjamini, Y. \& Hochberg, Y. (2000) On the Adaptive Control of the False Discovery Rate in Multiple Testing With Independent Statistics. Journal of Educational and Behavioral Statistics, 25, 60-83.

Brown, J.H. \& Kodric-Brown, A. (1977) Turnover Rates in Insular Biogeography: Effect of Immigration on Extinction. Ecology, 58, 445-449.

Chesson, P. (2000) Mechanisms of maintenance of species diversity. Annual Review of Ecology and Systematics, 31, 343-366.

Clark, J.S., Bell, D., Chu, C., Courbaud, B., Dietze, M., Hersh, M., HilleRisLambers, J., Ibáñez, I., LaDeau, S., McMahon, S., Metcalf, J., Mohan, J., Moran, E., Pangle, L., Pearson, S., Salk, C., Shen, Z., Valle, D. \& Wyckoff, P. (2010) High-dimensional coexistence based on individual variation: a synthesis of evidence. Ecological Monographs, 80, 569-608.

Collins, J.P. \& Storfer, A. (2003) Global amphibian declines: sorting the hypotheses. Diversity and Distributions, 9, 89-98.

Comita, L.S., Muller-Landau, H.C., Aguilar, S. \& Hubbell, S.P. (2010) Asymmetric density dependence shapes species abundances in a tropical tree community. Science, 329, $330-$ 332.

Freckleton, R.P., Watkinson, A.R., Green, R.E. \& Sutherland, W.J. (2006) Census error and the 
detection of density dependence. Journal of Animal Ecology, 75, 837-851.

397

398

399

Harpole, W.S. \& Suding, K.N. (2007) Frequency $\square$ dependence stabilizes competitive interactions among four annual plants. Ecology Letters, 10, 1164-1169.

Johnson, D.J., Beaulieu, W.T., Bever, J.D. \& Clay, K. (2012) Conspecific Negative Density Dependence and Forest Diversity. Science, 336, 904-907.

Knape, J. \& de Valpine, P. (2012) Are patterns of density dependence in the Global Population Dynamics Database driven by uncertainty about population abundance? Ecology Letters, 15, 17-23.

Kobe, R.K. \& Vriesendorp, C.F. (2011) Conspecific density dependence in seedlings varies with species shade tolerance in a wet tropical forest. Ecology Letters, 14, 503-510.

Kunin, W.E. \& Gaston, K.J. (1997) The Biology of Rarity: Causes and Consequences of RareCommon Differences, Springer.

Magurran, A.E. \& Henderson, P.A. (2003) Explaining the excess of rare species in natural species abundance distributions. Nature, 422, 714-716.

Main, A.R. (1982) Rare species: precious or dross? Species at risk: Research in Australia (ed. by R.H. Groves) and W.D.L. Ride), pp. 163-174. Australian Academy of Science, Canberra.

Mangan, S.A., Schnitzer, S.A., Herre, E.A., Mack, K.M.L., Valencia, M.C., Sanchez, E.I. \& Bever, J.D. (2010) Negative plant-soil feedback predicts tree-species relative abundance in a tropical forest. Nature, 466, 752-755.

Preston, F.W. (1948) The commoness and rarity of species. Ecology, 29, 254-283.

R Development Core Team (2012) R: A language and environment for statistical computing., $\mathrm{R}$ Foundation for Statistical Computing, Vienna, Austria.

Strimmer, K. (2008) A unified approach to false discovery rate estimation. BMC Bioinformatics, 9, 303.

Tilman, D. (2004) Niche tradeoffs, neutrality, and community structure: A stochastic theory of resource competition, invasion, and community assembly. Proceedings of the National Academy of Sciences of the United States of America, 101, 10854-10861.

Verhoeven, K.J.F., Simonsen, K.L. \& McIntyre, L.M. (2005) Implementing false discovery rate control: increasing your power. Oikos, 108, 643-647.

Xu, M., Wang, Y. \& Yu, S. (2015) Conspecific negative density dependence decreases with increasing species abundance. Ecosphere, 6, 1-11. 

not certified by peer review) is the author/funder. All rights reserved. No reuse allowed without permission.

427 Yenni, G., Adler, P.B. \& Ernest, S.K.M. (2012) Strong self-limitation promotes the persistence of 428 rare species. Ecology, 93, 456-461.

429 


\section{Biosketches}

431 Glenda Yenni is a multidisciplinary ecologist interested in drawing connections between

432 theory and observation in community ecology. Peter Adler is a plant ecologist interested in

433 explaining population and community dynamics in space and time. S.K. Morgan Ernest is a

434 macroecologist and community ecologist studying the temporal dynamics of community

435 assembly.

436 
437 Table 1: Summary results of community-level analyses by taxonomic group.

\begin{tabular}{|c|c|c|c|c|c|c|c|c|}
\hline & & $\mathrm{Me}$ & in Values & Across & Communit & Les & & \\
\hline $\begin{array}{c}\text { Taxonomi } \\
\text { c } \\
\text { Group }\end{array}$ & $\begin{array}{l}\text { Num. } \\
\text { communi } \\
\text { ties }\end{array}$ & $\begin{array}{l}\text { Spp/ } \\
\text { commu } \\
\text { nity }\end{array}$ & $\begin{array}{l}\text { Persis } \\
\text { tent } \\
\text { spp/ } \\
\text { commun } \\
\text { ity }\end{array}$ & $\begin{array}{l}\text { Observ } \\
\text { ed } \\
\text { covari } \\
\text { ance } \\
(\rho)\end{array}$ & $\begin{array}{c}\text { Random } \\
\text { ized } \\
\text { covari } \\
\text { ance } \\
\left(\rho_{0}\right)\end{array}$ & $\begin{array}{c}\text { Covari } \\
\text { ance } \\
\text { streng } \\
\text { th } \\
(\rho / \\
\left.\rho_{0}\right)\end{array}$ & $\begin{array}{c}\text { Num. } \\
\text { signifi } \\
\text { cant } \\
\text { communi } \\
\text { ties }\end{array}$ & $\begin{array}{l}\text { Proport } \\
\text { ion } \\
\text { signifi } \\
\text { cant. }\end{array}$ \\
\hline Birds & 14 & 25.9 & 21.1 & -1.68 & -1.29 & 1.38 & 7 & 0.50 \\
\hline Fish & 6 & 8.8 & 7.0 & -9.02 & -7.41 & 1.40 & 3 & 0.50 \\
\hline $\begin{array}{l}\text { Herpetof } \\
\text { auna }\end{array}$ & 11 & 11.4 & 7.0 & -0.41 & -0.67 & 0.27 & 1 & 0.09 \\
\hline $\begin{array}{l}\text { Inverteb } \\
\text { rates }\end{array}$ & 25 & 42.0 & 12.2 & -3.86 & -2.84 & 1.69 & 12 & 0.48 \\
\hline Mammals & 17 & 7.8 & 4.6 & -1.70 & -1.08 & 2.65 & 9 & 0.53 \\
\hline Plants & 17 & 82.0 & 22.6 & -3.74 & -2.61 & 1.64 & 9 & 0.53 \\
\hline Total & 90 & & & & & & 41 & 0.46 \\
\hline
\end{tabular}

438 Covariances $\left(\rho\right.$ and $\left.\rho_{0}\right)$ were calculated as $\operatorname{cov}(\log (\boldsymbol{f}), \log (-\boldsymbol{F D}))$. The number of significant 439 communities are those in which the covariance was determined significant after false discovery 440 rate control $(\alpha=0.1)$. 


\section{Figure Legends}

443 Figure 1: A) Negative frequency dependence and equilibrium frequency can be calculated from

444 time-series community data. Each data point represents the relative abundance of species $i$ at

445 time $t$ in its community (x-axis) and its log per capita population growth rate from time $t$ to time

$446 t+1$ (y-axis). The slope of this line is the species' negative frequency dependence $\left(N F D=-\beta_{1}\right.$ in

447 the linear model). Equilibrium frequency - the expected relative abundance of species $\mathrm{i}$ in the

448 community when its population growth is at equilibrium - is the fitted x-intercept of the

449 relationship. The fitted y-intercept is the species' maximal growth rate. B) The $\mathrm{x}$-intercept and

450 slope of the relationship indicate important biological information about each species. Steep

451 slopes indicate stronger negative frequency dependence, and thus higher sensitivity of population

452 growth to changes in conspecifics than to changes in heterospecifics. In a linear relationship,

453 high maximal growth rates, which buffer species from extinction, are achieved through high

454 equilibrium frequencies (common species, solid grey line), or strong negative frequency

455 dependence (rare species, solid black line). X-intercepts $<0$ indicate species that cannot maintain

456 a stable presence within the community at their population equilibrium. Species exhibiting

457 positive frequency dependence will often have difficulty invading when rare. These latter two

458 scenarios indicate species that are likely not stably coexisting within the current community.

459 Figure 2: An example of our analyses using the Portal rodent community. (A) The relationship

460 between relative abundance and growth rate is fit for each species. The slope of this relationship

461 is the "Strength of NFD" for each species, and the x-intercept is the "Equilibrium Frequency."

462 (B) Using each species as a datapoint, the covariance between these two parameters (-1.17 in this

463 example) is the value of interest, but it contains known biases. Randomizations of the time series

464 were used to create a baseline expectation for each unique community, and the observed 
465 relationship was considered relative to this baseline (the Randomized Covariance, -0.46 in this

466 example). Thus, we use the proportional difference in the empirical covariance from random

467 (observed covariance/mean randomized covariance, $-1.17 /-0.46=2.54$ ). The empirical pattern in

468 this community (solid black line, with shaded 95\% confidence intervals) compared to one

469 realization from the randomized results (out of 5000 randomizations), accounts for the bias in the

470 negative frequency dependence estimates introduced by measurement error and the artificial

471 relationship this creates (dashed line, with shaded 95\% confidence intervals). (C) P-values were

472 calculated as the proportion of randomized values larger than the observed asymmetry value (-

$4731.17, \mathrm{p}$-value $=0.0032$ in this example).

474 Figure 3: Results were compared to randomized data to account for bias introduced by

475 measurement error and determine which relationships were significant (colored bars) or not (grey

476 bars). P-values were calculated as the proportion of randomized values larger than the observed

477 asymmetry value. (A) All results for the 90 communities included in the analysis. We detected a

478 significantly asymmetric negative frequency dependence pattern in $46 \%$ of these communities.

479 (B) Results separated by taxonomic group, showing the relative size of the asymmetry in

480 negative frequency dependence between species in each community (Observed

481 Covariance/Randomized Covariance). The proportion value in each panel is the proportion of

482 communities in that group with a significant asymmetric negative frequency dependence pattern. 
(A)

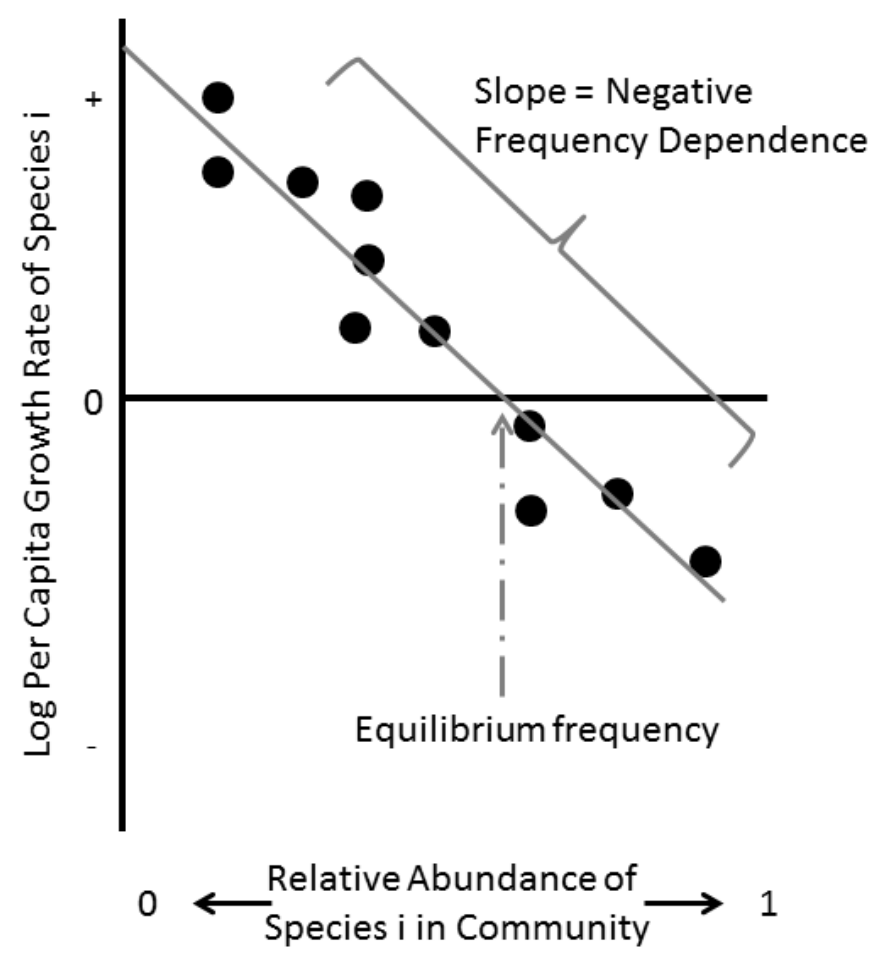

(B)

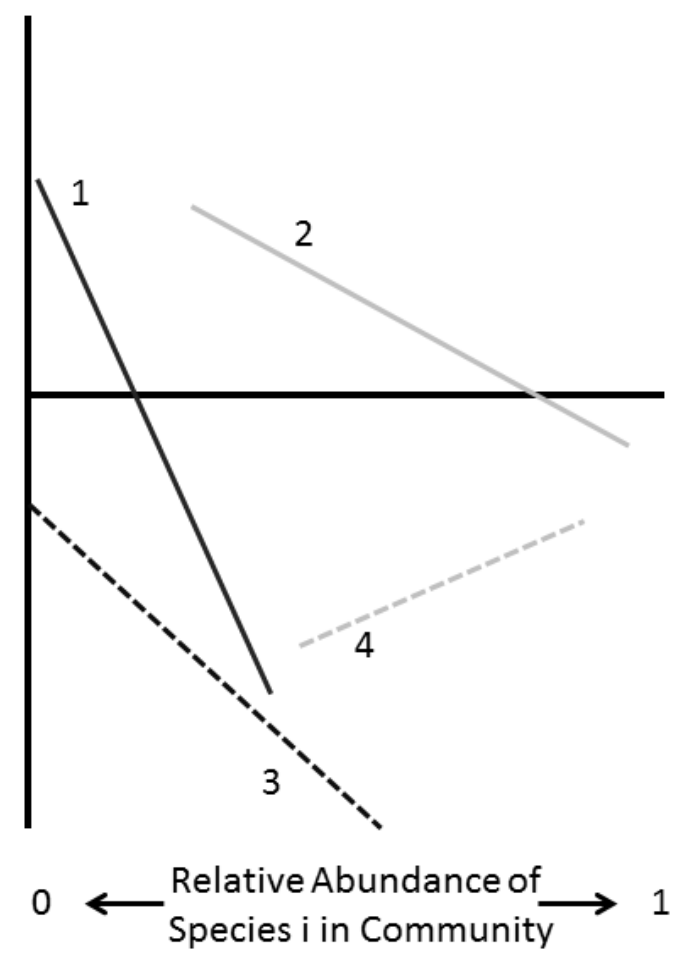



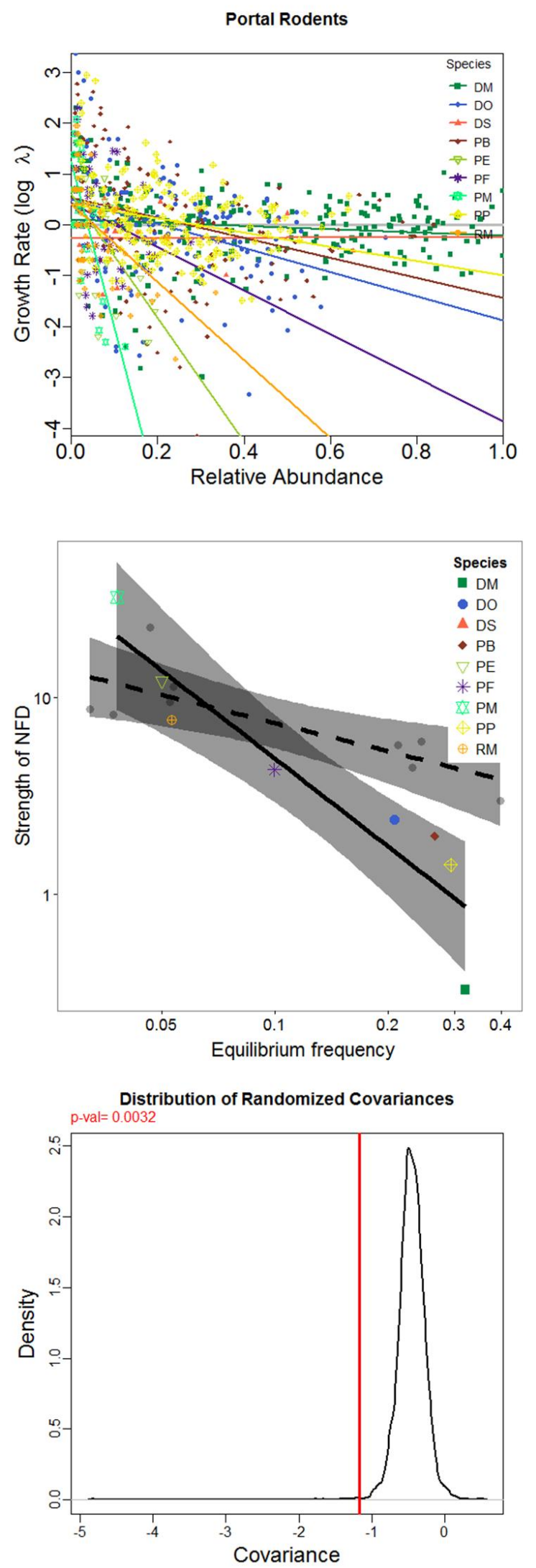

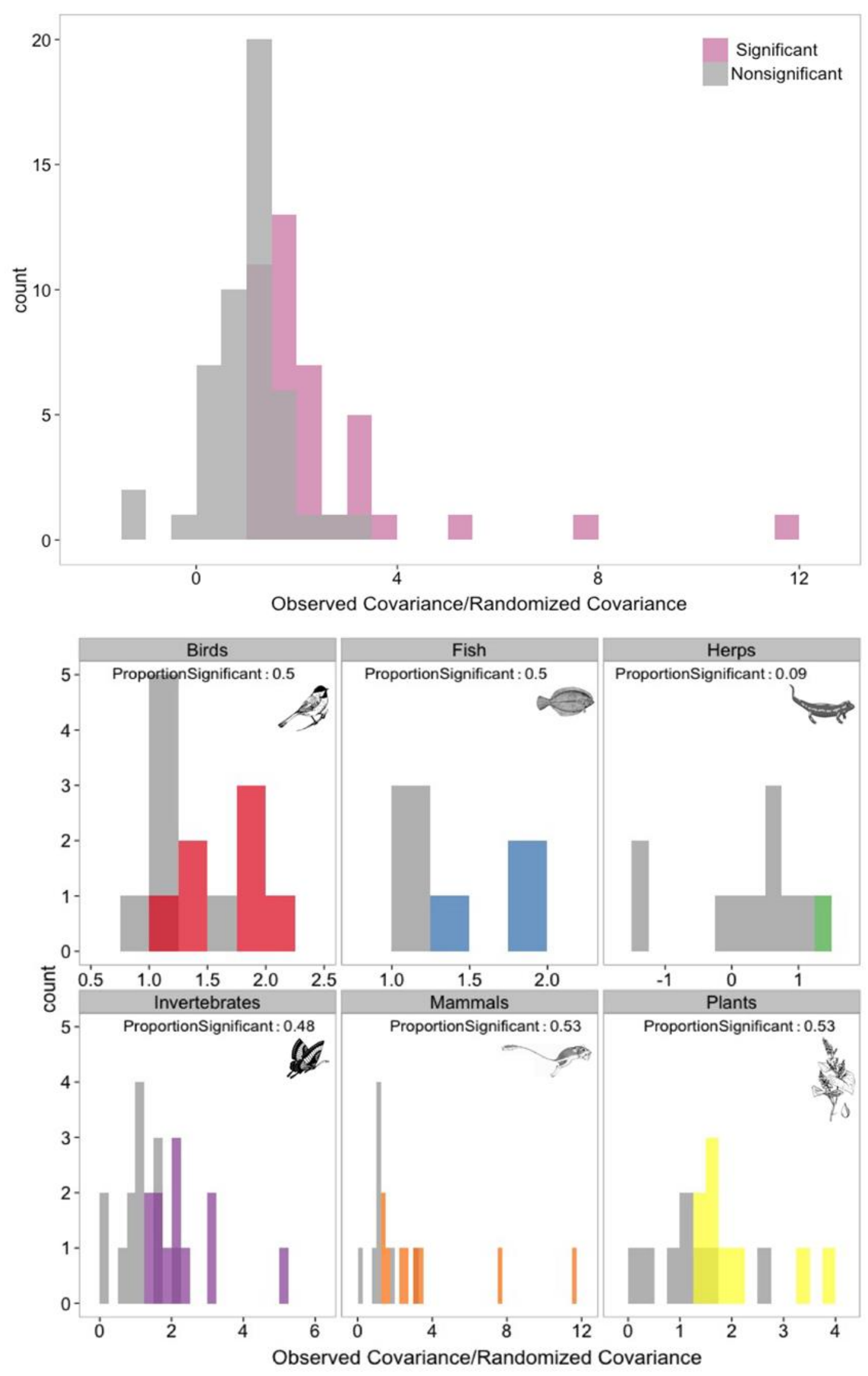